\title{
Combine Common Language with Dialect, Promote the Popularization of Chinese Phonology
}

\author{
Xiao Jiugen", Xie Lu \\ Research Center of Language and Language Life, Jiangxi Normal University, Nanchang, China \\ Email address: \\ jxsdxjg666666@sina.com (Xiao Jiugen), nine8765@qq.com (Xie Lu) \\ ${ }^{*}$ Corresponding author
}

To cite this article:

Xiao Jiugen, Xie Lu. Combine Common Language with Dialect Popularize Chinese Phonology. Science Innovation. Vol. 8, No. 5, 2020, pp. 144-149. doi: 10.11648/j.si.20200805.15

Received: September 27, 2020; Accepted: October 30, 2020; Published: November 4, 2020

\begin{abstract}
Chinese Phonology is a traditional and basic subject, which plays an important role in the study of characters, exegesis, ancient books. Chinese Phonology is also a compulsory basic course for postgraduates majoring in linguistics and philology. In the formation and development of this discipline, it contains both the essence of science and miscellaneous dross. Due to the particularity of the subject contents, the limitations of people's knowledge, and the inefficiency of teaching and learning methods, people are discouraged and few are willing to learn. Faced with this situation, if we want to carry on the phonological teaching and learning, teachers and students must study all kinds of effective methods. One of the most practical and effective methods is that taking the pronunciation of Mandarin as reference and taking the pronunciation of Dialect as evidence. Our specific approach is based on the content of phonology, guided by the theory of Mandarin, oriented by problems, and supplemented by the cases of Dialect, and the relationship and corresponding laws among them will be explained. Demonstrating the past with today's idea, mutual confirmation between ancient and present, this not only makes obscure and complex content easy to understand, but also achieves a commendable effect. Therefore, as long as the teachers and students adhere to acquire new knowledge while thinking over the old, and get rid of deficiency by practicability, it will be possible to restore the true features of this discipline.
\end{abstract}

Keywords: Chinese Phonology, Teaching, Learning, Mandarin, Dialect, Popularization

\section{牵引共同语和方言，促使汉语音韵通俗化}

\section{肖九根 ${ }^{*}$, 谢璐}

江西师范大学语言与语言生活研究中心, 南昌, 中国

\section{邮箱}

jxsdxjg666666@sina.com（肖九根）, nine8765@qq.com（谢璐）

摘要: 汉语音韵是一个传统的基础学科, 它对文字、训诂、古籍经典以及方言等学习与研究有着重要作用, 也是语言 文字学等专业研究生必修的基础课。在这一学科形成与发展过程中, 其内容既有精当而科学的精华, 也有繁杂而欠当 的糟粕。由于学科内容的特殊性、人们认识的局限性、教学与学习方法的失灵性等原因, 致使人们望之却步, 愿习之 者甚少。面对此种情形, 如要进行音韵教学与学习, 教者与学者定要研究各种行之有效的方法。在诸多方法中, 以普 音作参照、方音为佐证是最实用、最有效的方法之一。我们的具体方法是：以音韵内容为依据，以普音理论为指导， 以问题为导向, 并辅之以方音案例, 阐释三者之间的相互关系与对应规律, 这样以今证古, 古今互证, 不仅使晦涩复 杂的内容通俗易懂, 而且还取得了很好的效果。所以, 音韵教者与学者只要坚持以溯源、温故求其新, 以实用、浅出 去其虚,还原学科的本来面目指日可待。 
关键词: 汉语音韵, 教学, 学习, 普通话, 方言, 通俗化

\section{1. 引言}

汉语音韵教学与学习方法问题, 是研究生专业基础 课中一个值得探讨的重要问题。之所以是一个值得探讨 的重要问题, 因为这关系到这个学科教学与学习的成功 与否。

关于汉语音韵教学与学习方法问题的讨论, 虽然有 一些见之于学术论文或散见于有关专著的相关内容之中, 但就其如何结合民族共同语——普通话（以下简称“普 音”）和地域交际语——方言（以下简称“方音”）来进行 教学与学习的研究成果不多。以笔者陃见, 有的专家学 者尽管谈到音韵与普音或音韵与方音问题, 而谈的是其 相互关系[1]或方言区如何利用音韵、方音分辨普音平、 趐声母读音问题 [2]。即使有谈教学与学习方法问题, 如 任晓肜（2011）[3]、陈大为（2012）[4]、陈会兵（2013） [5]、尹喜清（2016）[6]、周映映（2019）[7]和裴梦苏 (2020) [8]等, 针对的是本科层次古汉语或古诗文教学 与学习问题, 而非更高层次 (如研究生) 将汉语音韵作 为一个学科体系来谈教学与学习方法的, 因而其研究虽 有理论意义和实践价值, 但其局限性也是显而易见的。 而真正面对研究生这一层次谈汉语音韵教学与学习方法 问题的, 有邹晓丽和胡佳佳等学者。邹晓丽在其学术论 文 (1999) [9]和专著（2002）[10]（p10-12）中均以较大 篇幅阐述了科学而正确学习的态度以及教学方法问题, 胡佳佳 (2018) [11]则以现代网络分析法谈其在音韵教学 与学习中的应用问题。毫无疑问, 其研究不仅具有方法 论的意义, 还有实际参考价值。不过有些遗憾的是, 汉 语音韵教学与学习如何结合普音与方音这样具体而实际 的问题, 至今还鲜有专家学者发表高论。鉴于这一点, 现在我们就这一问题作些探讨, 这不仅对于提高研究生 的音韵教学效果与学习效率将会产生较大的作用, 而且 在方法论方面也具有重要的理论意义和实际应用价值。

\section{2. 汉语音韵在其形成与发展过程中具有一定的 古奥性和复杂性}

汉语音韵作为一个传统学科, 运用历史语言学方法 以研究汉语肇始于 20 世纪初。但是, 这一学科从魏晋时 期 (即公元 2 世纪反切盛行之际) 发轫至今, 已有 1800 多 年的历史了。也就是说, 远在魏晋南北朝时期, 中国学 者就开始了汉语音韵的专门研究, 而且其时的研究达到 了颜之推所描述的“音韵锋出”那种盛况[12]（p230）。在 其研究过程中, 汉语音韵这一学科也随之建立起来了, 这个学科发展迄今也有一千多年历史了。后来, 人们将 汉语音韵与文字、训诂总称之为语言文字的传统学科一 —“小学”。

在汉语音韵研究与发展中, 古音韵学家不仅建立了 古音学 (即上古音) 、今音学 (即中古音) 、北音学 (即近古音) 等不同历史时期的音理知识体系, 而且还
将其绘制成若干图表, 以之分析声韵调结构, 阐述发音 原理与方法, 进而建立了研究汉语音理的等韵学。

在其形成与发展的过程中, 汉语音韵这一学科的内 容既有精当而科学的精华, 也有繁杂而欠当的糟粕。言 其内容精当而科学, 诸如它以反切注音、以声韵调音类 推究相互对应关系及古今流变规律、以诗辞曲韵律构拟 历代声韵音值、以系联法考证古声韵分类等内容, 这些 既是今天我们教学、学习、研究的精华, 也是我们承继、 运用的主要理论方法。言其内容繁杂而欠当: 一是受历 史时代局限, 古音韵没有现代语音学理论作指导, 研究 声母无法从物理生理属性去描写其发音部位和发音方法, 更无从将其音值构拟出来; 研究韵母仅以“韵摄”“韵 部”“小韵”等为单位, 无法从韵头、韵腹、韵尾去准确地 分析其组织结构; 研究声调只有从调类入手, 其高低升 降变化无法通过调值精确地表达出来。二是传统古音学、 今音学和等韵学不少内容及称名阐释, 不仅不科学或不 严密, 还十分繁杂而晦涩, 如把声母发音部位“唇舌齿牙 喉”比附于音乐“宫商角徵羽”, 有的将之依附于方位“东 西南北中”, 把声母发音方法如送气不送气的塞音、塞擦 音及擦音、鼻音、边音称之为“戛透拂捺炼”, 且其不同 学者对同一声母归类也出现了很多相悖现象; 有的学者 把声调释之为“平声锃铭, 上声苍老, 去声脆嫩, 入声直 朴”[13](p9), 甚至还将“平上去入”的读法喻之为“钟鼓罄 柷”, 如此这般, 不遑而论。这无疑给教学、学习、研究 者带来了极大的困惑。三是从其产生那时起, 汉语音韵 就置于特定的哲学思想控制之下, 完全臣服于古代那种 礼乐教化, 这使其内容渗透着一定的思想观念, 使其科 学性蒙上了一层玄乎其玄的神秘面纱, 这是汉语音韵被 人视为“绝学”的重要原因之一。当然, 还有一些其它方 面的问题, 如汉语音韵不少术语完全搬用梵文, 未加任 何改造就直接用于汉语音韵研究, 以致造成概念意义含 混玄虚、音韵音理描述不清、声韵母分类不准等问题, 而这正是汉语音韵难懂之处。正是存在这样或那样晦涩 难懂的问题, 致使汉语音韵不单一般人谈之色变, 望而 却步, 就连一些大学者也为之兴叹。例如, 清·段玉裁清 楚韵部支、脂、之在先秦两汉时是三个不同的韵类, 但 不清楚这三个韵部为何这样分, 其音值究竟为何, 所以 他写信向江有诰求教: “足下......能确知所以支脂之分为 三之本源乎？......仆老耄, 倘得闻而死, 岂非大幸也! ”

(段玉裁《答江晋三论韵书》) 另一学者戴震知道先秦 存有阴、阳、入三类韵部, 但其韵部音值有何差别, 无 从知晓, 认为阳声“犹击金成声也”, 阴声“犹击石成声 也”, 由于认识上的含混, 以致在分析具体音韵时就出现 了错误, 如把阴声韵当成了阳声韵。就是近代著名语言 文字学家、国学大师黄㑆也说: “音韵学之至, 不能以口, 不能以耳, 则以心知。故口得者半, 耳得者半, 心得者 亦半。犹三十六母知照、彻穿之不分, 唐以前以后读皆 同耳, 就《唐韵》知之, 吾口耳不能分, 独心能分之 耳。”[14] (p26) 
诚然, 有的问题是语音发展变化所产生的, 因为时有 古今, 地有南北, 所以音也发生了转移。例如, 古汉语 “玄、黄”声纽皆为全浊匣母 $\gamma$, 属于双声, 而普音已非双 声; “剑、阁”皆为见母 $\mathrm{k}$, 亦双声, 今粤方言还属双声 $\mathrm{k}$, 而普音则非双声, 这是语音演变的结果。因此, 学习、研 究汉语音韵, 还要有“时”“地”观念。

学习、研究汉语音韵有多方面的作用, 它对于文字 学、训诂学的学习与研究以及古籍经典阅读作用极大。 关于这一点, 清代学者段玉裁曾经说过: “音均（韵）明 而六书明, 六书明而古经传无不可通。”[15]（p805）此 外, 汉语音韵不仅对现代汉语学习与研究、方言调查与 研究等有十分重要的作用, 甚至还对中国历史、文化、 音乐、美术、戏曲等方面的学习和研究也有帮助。

现在的问题是：如何将这个学科传承下去，使之后 继有人, 并在新时代发挥它应有的作用。毫无疑问, 其 问题的关键就在于汉语音韵教学与学习, 解决这个问题 的钥匙就掌握在汉语音韵教者与学者的手中。在汉语音 韵教学与学习过程中, 教者与学者如能有的放矢, 善于 将其深奥复杂的内容化为简单通俗易懂的理论知识, 而 不是一味地去纠缠那些过时的杂而无用、䀲涩难懂的内 容及称名术语。汉语音韵有其独特性, 其独特之处就在 于它是“口耳之学”。学者惟有“因声而听或因听而思”, 才能“由因听而思进入最后的因听而悟”，而“由思到悟是 一个突进过程”, “意味着思想冲破壅塞后豁然开朗, 于 刹那间绽放出灿烂的火花”, 这是质的飞跃, 新的境界 [16]。于是, 学者学之有感, 学之有得, 学之有用。在诸 多方法中, 将汉语音韵与普音和方音结合起来, 是其重 要而有效的教学与学习方法之一。

\section{3. 汉语音韵与普音和方音有着天然的渊源共生 关系}

民族共同语一一普音是汉语音韵的一脉传承，而地 域交际语一一方音又是汉语音韵的直接派生。

以汉语音韵与普音而言, 它们是源与流的关系。例 如, 普音中的声母 $\mathrm{b} 、 \mathrm{p}$ 就是来源于古音韵唇音“帮浐並” 三纽的，声母zh、ch、sh来源于古音韵舌上音“知彻澄”、 正齿音“庄初崇生”和“章昌船书禅”三组声纽，而声母 $\mathrm{j} 、 \mathrm{q}$ 、 $\mathrm{x}$ 则是由古音韵齿头音“精清从心邪”、牙音“见溪群”和喉 音“晓、匣”三组声纽腭化而成的。又如, 普音中韵母 $\mathrm{a}$ 的 来源非常广, 其来源有与端系声纽组合的果摄歌部开口 一等韵, 与端系、知系声纽组合的咸摄合、盍、洽部开 口一、二等韵, 与帮系、端系、知系声纽组合的山摄曷、 點部开口一、二等韵和月部合口三等韵, 还有与端系声 纽组合的梗摄庚部开口二等韵; 而韵母 $\mathrm{ia}$ 则来源于同见系 声纽组合的四个开口二等韵：蟹摄佳部、假摄麻部、咸 摄狎部和山摄鎋部; 韵母如ün源自与见系声纽组合的臻 摄合口三等韵这样来源单一的情况极少。普音中声调也 是源于古音韵四声，尽管变化较大，但也具有强而整齐 的对应规律: 平分阴阳, 浊上变去, 入派三声 (普音即 四声)。也就是说, 古音韵声调演变为普音声调是按其 声纽清浊条件进行分化的: 古音韵清声纽平声字今读普
音阴平, 其浊声纽平声字则读普音阳平; 古音韵清、次 浊声纽上声字, 普音仍读上声, 全浊声纽上声字今读去 声了; 古音韵全浊声纽入声字归为普音阳平, 次浊声纽 入声字今读作去声了。由此可见, 普音与古音韵的关系 十分密切。可以毫不夸张地说, 没有古音韵就没有当今 的民族共同语一一普通话。

就汉语音韵与方音来说, 它们之间存在着衍生分派 的关系。20世纪初叶, 瑞典汉学家高本汉 (B.Karlgren) 以历史比较法研究汉语与方音的关系, 认为“汉语的演变 从上古到中古再到现代汉语方言是直线发展的, 由周秦 古音(《诗经》音系谐声音系)演变为隋唐《切韵》音系, 又从《切韵》音系中分化出现代汉语各个方言音系”[17] (p27)。他“以《切韵》为枢纽, 上联先秦古音, 下联 现实的方言, 把文献资料和方言的差异结合起来, 整理 和解释汉语演变的规律。《中国音韵学学研究》就是这 一理论体系的核心和基础, 集中研究方言和《切韵》的 关系。他认为切韵音系是现代汉语各个方言的原始母 语......”[18]（p233）。王力也认为汉语各方言“同出一 源”, 是“兄弟姊妹”的关系。他说: “汉语方言是历史形 成的。各地的方言无论如何复杂, 追溯到最后还是同出 一源。这就说明了为什么各地方言的语音有着对应的规 律...... 从历史上看方言的发展......原来这些方言都是兄 弟姊妹, 各自保存着老祖宗的一些东西。”[19]（p685686）另外, 游汝杰也有过类似的论述, 他说: “汉语南 方六大方言：吴、湘、粤、闽、赣、客的产生, 都是由 历代北方居民向南方迁徙发其端的。”[20]（p47）“汉语 南方方言吴语、湘语、粤语、赣语、平话的直接源头是 古代北方汉语, 闽语和徽语是从吴语分化出来的, 客家 方言是从赣语分化出来的。”[21]（p110）并在《中国文 化语言学引论》中再次作过类似的重申[22]（p24）, 而 且还描画出汉语方言起源与分化的示意图:

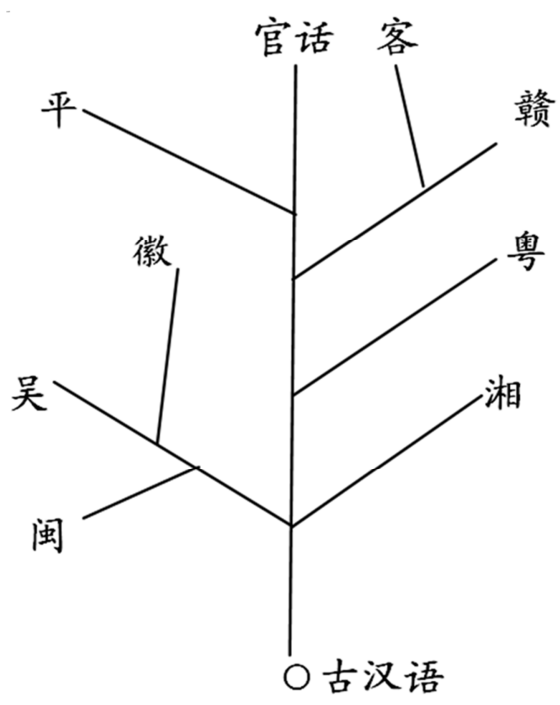

图1 汉语方言起源与分化。

从树形图上可以看出, 南方各大汉语方言是同一母 语古汉语派生出来的子孙后代。

对于这种汉语方言的发展观, 有的学者并不完全认 同。邓晓华认为南方方言的形成既非完全“土生土长”, 
也非完全“北方迁入”, 是一个“多元结构体”, 如福建闽 越、广东南越、江西干越、江浙吴越等共同构成了一个 南方文化交互圈, 这是南方汉语方言形成的基础; 其次 历代南迁的北民与土著人杂居, 彼此形成了一种语言文 化互动机制, 从而形成了“复合型”或“混合型”的汉语方 言[23]（p279）。潘悟云从南方人类基因与北方汉民不一

\section{古代汉语}

A

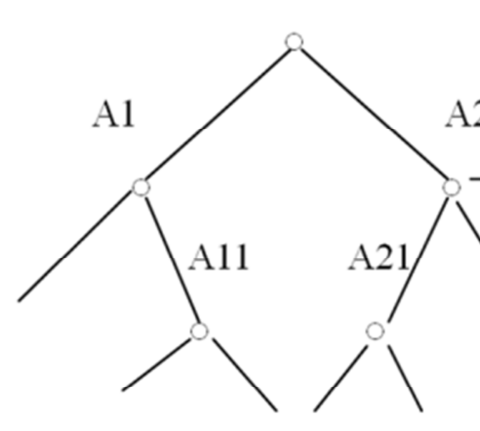

现代北方方言
样这个角度论证南方方言形成的主体是土著居民而非北 方移民，只是他们在北方文化及语言不断影响下形成了 以本族语为底层的混合语一一南方汉语方言, 并得出结 论:现代南方汉语方言并不是从同一母语中分化来的, 而 是几种母语相互接触、相互混合的结果 [24]（p59-67)、 [25]（p298-313）。这种语言演变如图所示:

\section{古百越语}

$\mathrm{B}$

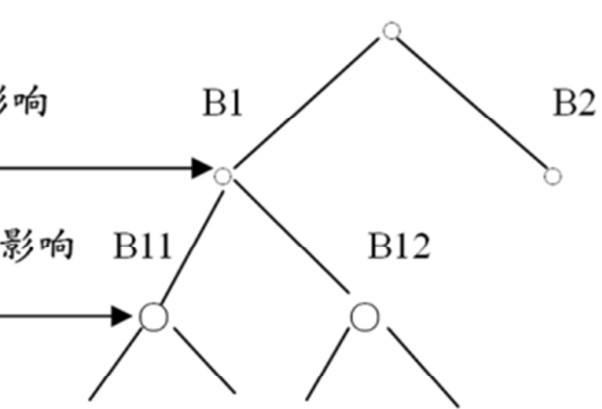

现代东南方言

（吴、湘、贑、客、曾、闽）

\section{语言接触、混合而形成新语言的模型}

图2 汉语方言演变模式。

不过, 无论如何, 汉语音韵与汉语方言有着十分密 切的关系, 这是不容否认的。否则, 今天的南方各大汉 语方言与中古音就不会有如此严整的对应规律。例如,

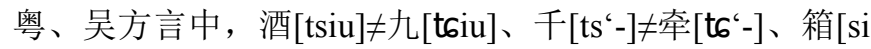
a ] ] 香[6ian]等读音不同, 前者还有存古性质, 仍读古音 韵的精组齿头音, 尚未腭化成舌面音。

一言以概之, 汉语音韵不仅是普音的直接源头, 还 与方音存有密切的源流共生关系, 它们之间有着整齐的 对应关系和严整的发展规律。

\section{4. 汉语音韵教学与学习紧密联系普音和方音所 产生的显著效果}

在汉语音韵教学与学习中, 教者与学者必须始终坚 持这一原则: 一手牵着民族共同语一一普音, 一手率着 地域交际语一一方音, 这样就会产生事半功倍的教学效 果。

众所周知，汉语音韵既是中文系本科专业选修课， 又是语言文字学专业研究生的必修课, 这一学科教学是 以古音(先秦音)、今音(隋唐音)、等韵和近代音一一历史 语音为对象, 以其历时变化为内容, 既要考察汉语音韵 的历史发展变化, 又要探寻其间的相互关系及演变规律。 因此, 其内容十分古奥, 关系极为复杂, 以致不少人“童 稚从事而皓首不能穷其理”[26]。由于学科内容的特殊性、 人们认识的局限性、教学与学习方法的失灵性等问题, 造成了学习者望之畏惧、见之裹步、愿习之者少的异常 现象。
而要扭转这一悖理局面, 音韵教师就不能完全沿袭 过去那套陈旧而繁琐的方法进行教学, 应该与时俱进, 采取更加适用而科学的现代教学方法, 设法为学生搭建 一个台阶, 创造一些条件, 使之通往汉语音韵彼岸之途 变得更平坦一些。

多年来，在音韵教学与学习中，我们一直采用以民 族共同语——普音为观照, 以地域交际语——方音为佐 证的方法, 这不仅破解了长期以来所面临的困境, 学生 不再有畏难情绪, 而且还取得了意想不到的效果, 其研 究成果也十分显著。例如, 每届的硕士研究生通过汉语音 韵理论知识的学习, 不但可以独立进行方言田野调查, 还 能运用其理论知识对方言音系及特点进行描写与分析, 并 以之作为学位论文选题进行研究。不仅如此, 还有不少 学生在国内外核心期刊上发表音韵方言研究方面的学术 论文。

关于汉语音韵的教学与学习方法, 我们的具体做法 是: 首先, 确定讲授、学习的汉语音韵理论知识点, 在 这一前提条件下，再以民族共同语——普音理论为指导， 以教学中设置的问题为向导, 并辅之以各方言区的方音 案例, 阐释音韵同普音和方音的相互关系与对应规律。 例如, 为了讲解古音韵尾问题, 教师先提出问题: “从中 古音到普通话语音的韵尾系统发生了较大的变化。请问 二者韵尾有哪些相同点和不同点? ”并给予学生一定的时 间进行思考。之后, 再引进普音韵尾理论, 请学生作比 较并解释说明, 其结论是: 普音韵尾系统在继承中古音 的基础上有所简化。也就是说, 中古音、普音阴声韵二 者均有开韵尾和元音尾; 中古音阳声韵尾有前鼻音-n、 
后鼻音- $n$ 和双唇鼻音- $m$, 而普音仅有前、后两个鼻韵尾, 没有双唇鼻韵尾; 中古音有入声塞韵尾-p、-t、- $\mathrm{k}$, 而普 音则已消失。继之, 以方音案例证其存古性。例如, 南 方不少方言阳声韵除与普音一样有前、后鼻韵尾外, 还 有双唇鼻韵尾-m, 另外还有入声塞韵尾（仅举普音中没 有的例子，声调从省）：粤方言广州话“暗nem、蓝lam, 目tep、罟ep, 嗐hat、抈iøt, 索 sak、力lik”, 南宁话“男 nam、蓝lam, 接tsip、怯hip, 法fat、辣lat, 墨mək、落 lək”; 客方言梅县话“三sam、柑 $k a m$, 叶iap、贴tiap, 热 niat、末 未at, 角kok、粟siuk”; 闽方言厦门话“啉lim、嘇 sam, 贴 tiap、廿liap, 不put、物but, 局kiJk、约iJk”。 相较而言, 赣方言有些不一样, 它没有双唇鼻韵尾, 入 声塞韵尾也不完整, 南昌、新建、永修等地入声塞韵尾 仅存- $\mathrm{t}$, 韵尾-p已与-t合而为一了, 而塞韵尾- $\mathrm{k}$ 则演变为 由入声韵转化为阴声韵的过渡阶段喉音尾-?了, 如南昌 话“八 bat、杂 tsat (中古音-p尾) 、烛 tsu? (中古音-k 尾)”。还有这样的情况, 共同语-普通话属同音的, 方 言不一定同音，如“立、栗、力”这三字，普通话同音而 粤方言广州话则不同音，它们分别读作“lap、løt、1 Ik”。 由此可见, 以这样的方法进行汉语音韵教学与学习, 这 就起到了四两拨千斤的神奇效果, 学生不单懂得了音韵 原理, 更为重要的还学会了运用音韵原理进行研究了, 不再存在过去那种谈之色变的现象了。

又如, 音韵尖团音也是一个重点、难点问题。就一 般情况而言, 学生不仅难以分析这一问题, 甚至要透彻 理解这个问题都很困难, 更谈不上去运用它了。为了解 决这一问题，教师特别要讲清楚其变化规律：中古音“精” 组声纽变化是以今普音韵母四呼的配合为变化条件的, 如“精”组声纽与普音开口呼（即古开一、二等韵）、合 口呼（即古合一、二等韵）组合，今仍读尖音 $\mathrm{ts}$ 、 ts'、 $\mathrm{s}$; 如与普音齐齿呼（即古开三、四等韵）、撮呼（即古合 三、四等韵）组合，则与中古开、合三、四等韵的“见、 晓”组声纽合而为一, 均腭化为团音 $6 、$ t 6 “ $、 6$ 了。关于这 一点, 方言区的不少方音至今还分尖团音, 如广州、安

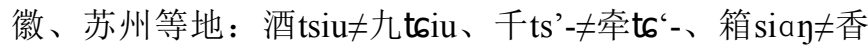
6ian等; 更有趣的是, 有人居然把“双喜6i临门”说成“双 死sil临门”。有的方言区, 中古“见、晓”组声纽即使与开、 合三、四等韵组合, 其读音也未发生腭化, 还存古音痕 迹。如曾经有个北方人在广州买甘蔗, 卖蔗的人说: “一 角钱一 kan55。”北方人以为是“一角钱一根”, 付了一角 钱拿起一根就走。可那人赶上来夺过甘蔗大叫起来: “一 根甘蔗有好几kan55! ”北方人这才恍然大悟: “啊, 是一 角钱一斤哪！“斦”普音t6in55, 与“根”ken55读音完全不 同, 而广州话“斤、根”音近, 皆为开口呼。也就是说,

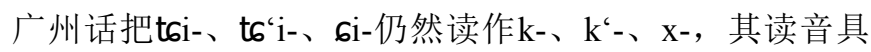
有鲜明的存古性质。教学音韵充分利用这些有利条件, 学生往往豁然开朗, 理解起来很容易。

所以, 教授和学习汉语音韵时不能就其本身之论而 论, 而应引入共同语一一普音的声韵理论, 还要辅之以 方音案例, 以今证古, 古今互证, 并引导学生根据所提 问题将其比较, 找出其间的共存关系与严整规律, 这使 学生有一种峰回路转、柳暗花明、似曾相识的奇妙感觉。
如此一来, 他们不仅知其然, 还知其所以然, 印象特别 深刻, 运用起来也会得心应手。

\section{5. 结语}

总而言之, 在研究生的音韵教学与学习过程中, 为 了揭开这 “绝学”的神秘面纱, 认真研究其教学与学习方 法不仅显得十分必要, 而且意义非凡。诚然, 适用音韵 教学与学习的方法多种多样, 而以普音作其参照、方音 为其佐证的方法则是诸多方法中最实用、最有效的方法 之一。只要我们紧紧牵引普音和方音这“两头”颈领, 就 能使晦涩难懂、纷繁复杂的汉语音韵理论化为通俗易懂、 一学就会的一般内容。

从汉语音韵与普音的关系来说, 正如有的学者所说 的普音是汉语音韵发展的必然结果, 其声韵调格局整齐, 不仅符合语音学“一音一符”音素化原则, 而且还符合音 韵学即传统音位学原理[1]。所以, 只要我们凭借已经熟 练掌握的普音基础理论, 就可以溯其源, 探其流, 寻其 律, 这样由易而难、由简单至复杂、由已知求未知, 逐 层推进, 习斯音韵何难之有?

以汉语音韵与方音的关系而言, 它们就像是一根藤 上结的瓜, 剪不断, 理还乱。正因为二者关系密切, 上 世纪末有学者主张建立一门新的交叉学科——方言音韵 学, 认为方言研究以音韵为指导, 音韵研究以方言为佐 证, 二者结合起来研究已显示出无可替代的优越性[27]。 实践证明, 汉语音韵教学与学习如将二者结合起来, 同 样相得益彰, 效果显而易见。

因此, 在音韵教学与学习中, 只要我们一手领着普 音, 一手牵着方音, 以溯源、温故求其新, 以实用、浅 出去其虚[10]（p10-12）, 不偏不倚, 还原音韵学科本来 面目就指日可待。在不久的将来, 汉语音韵学科理论定 会成为研究生学子们日常品尝的美馔佳有。

\section{致谢}

基金项目: 江西省学位与研究生教育教学改革研究 项目“揭开“绝学”面纱, 继承传统文化一一关于研究生课 程《音韵学》教改问题的几点思考”阶段性成果 (编号: JXYJG-2014-038）。

\section{参考文献}

[1] 唐作藩.从音韵学看汉语拼音方案的优越性[J].语言文字应 用2013(S1)。

[2] 黎新第.方言、音韵与普通话平、越舌声母字的分辨[J].重 庆师范大学学报2007(02)。

[3] 任晓肜.高等师范院校古代汉语音韵教学探索[J].内蒙古师 范大学学报(教科版)2011(09)。

[4] 陈大为. 高校古代汉语课程音韵学教学内容与方法改革初 探[J].吉林教育学院学报2012(06)。 
[5] 陈会兵. 《古代汉语》课程“音韵”部分教学方案探索 [J].重 庆三峡学院学报2013(06)。

[6] 尹喜清.论方言在音韵学教学中的运用[J].现代语文(学术 版)2016(09)。

[7] 周映映.谈从音韵切入古典诗词教学[J].语文教学之友 2019 (03)。

[8］裴梦苏.走出“绝学”困境——当下通行古代汉语教材中音韵 学内容编写策略研究 [J].长春师范大学学报2020（01）。

[9] 邹晓丽. 对讲授、研究传统音韵学的思考 [J].古汉语研究 1999(01)。

[10] 邹晓丽.传统音韵学实用教程 $[\mathrm{M}]$. 上海：上海辞书出版 社,2002。

[11] 胡佳佳.网络分析方法在音韵学教学中的应用——以广 韵》反切系联为例 [J].励耘语言学刊2018（02）。

[12] 晋·颜之推.颜氏家训译注[M]庄辉明, 章义和撰.上海: 上 海古籍出版社, 2012。

[13] 明·袁子让.字学元元 $[\mathrm{M}]$, 转引自刘晓英《<字学元元 $>$ 研 究》.长沙: 湖南师范大学出版社, 2017。

[14] 杨剑桥.汉语音韵学讲义[M].上海: 复旦大学出版社, 2005 。

[15] 清·段玉裁. 说文解字注[M].上海：上海古籍出版社，1981。

[16] 傅修延,邱宗珍.因声而听、因听而思和因听而悟——试论 闻声之作的三重境界[J].江西师范大学学报(哲社科版)2019 (02)。
[17] 李葆嘉.汉语起源与演化模式研究 $[\mathrm{M}]$. 哈尔滨: 黑龙江教育 出版社, 2001。

[18] 徐通铭,陈保亚.二十世纪的中国历史语言学[A].刘坚.二十 世纪的中国语言学[M].北京: 北京大学出版社, 1998年。

[19] 王力.王力文集第三卷[M].济南：山东教育出版社，1985。

[20] 周振鹤, 游汝杰. 方言与中国文化 $[\mathrm{M}]$. 上海: 上海人民出版社, 1986 。

[21] 游汝杰.汉语方言学导论 [M].上海: 上海教育出版社, 1992 。

[22] 游汝杰.中国文化语言学引论[M]. 北京: 高等教育出版社, 1993。

[23] 邓晓华.试论古南方汉语的形成[A].邹嘉彦.语言接触论集 $[\mathrm{M}]$.上海:上海教育出版社, 2004。

[24] 潘悟云.汉语方言的历史层次及其类型[A].石锋.乐在其中王 士元教授70华诞庆祝文集 $[\mathrm{M}]$.天津：南开大学出版社, 2004。

[25] 潘悟云.语言接触与汉语南方方言的形成[A].邹嘉彦.语言接 触论集 $[\mathrm{M}]$.上海:上海教育出版社, 2004 。

[26] 董同䧻.汉语音韵学·序[M].北京:中华书局,2004。

[27] 殷焕先,张玉来,徐明轩. 方言音韵学构架 [J].山东师大学报 (社科版)1990(01)。 